\title{
Analogues of glacial valley profiles in particle mechanics and in cosmology
}

\author{
Valerio Faraoni $^{\mathrm{a} \star}$ and Adriana M. Cardini ${ }^{\mathrm{a}}$ \\ ${ }^{a}$ Physics Department, Bishop's University, 2600 College Street, Sherbrooke, QC J1M 1Z7, Canada \\ *vfaraoni@ubishops.ca
}

\begin{abstract}
An ordinary differential equation describing the transverse profiles of $U$-shaped glacial valleys has two formal analogies, which we explore in detail, bridging these different areas of research. First, an analogy with point particle mechanics completes the description of the solutions. Second, an analogy with the Friedmann equation of relativistic cosmology shows that the analogue of a glacial valley profile is a universe with a future singularity of interest in theoretical models of cosmology. A Big Freeze singularity, which was not previously observed for positive curvature index, is also contained in the dynamics.
\end{abstract}

Key words: glacial valley transverse profiles, glaciology-cosmology analogies, glaciology-mechanics analogies, Friedmann equation

\section{Introduction}

It has long been acknowledged in glaciology (since its inception) (Campbell 1865; McGee 1894), and it is common knowledge in elementary geography, that valleys carved by glaciers are U-shaped, whereas valleys carved by rivers are V-shaped. Here we focus on the former. The detailed and continued process of reshaping a valley by a glacier via erosion of the valley walls and floor over time is complex and is best modelled with numerical techniques (Harbor 1995; Seddik et al. 2009; Yang and Shi 2015). If one is interested only in the final result of the glacier action, simpler analytical approaches can be used. Given the scarcity of analytical models in the literature, theoretical approaches to this problem are valuable. Hirano and Aniya (1988) proposed a variational principle extremizing the friction of ice against the valley walls, subject to an appropriate constraint. Let the cross-sectional profile of a glacial valley be described by a function $y(x)$, where $x$ is a coordinate transverse to the glacier flow. Hirano and Aniya (1998) argued that friction (a functional of the cross-profile $y(x)$ ) should be minimal at the end of the erosion process, subject to the constraint that the contact length of the cross-profile of the ice is constant. This contact length between two endpoints $x_{1}$ and $x_{2}$ of the transverse profile is

$$
s[y(x)]=\int_{x_{1}}^{x_{2}} d s=\int_{x_{1}}^{x_{2}} \sqrt{d x^{2}+d y^{2}}=\int_{x_{1}}^{x_{2}} \sqrt{1+\left(y^{\prime}\right)^{2}} d x=\text { const. }
$$

work is licensed under a Creative Commons Attribution 4.0 International License (CC BY 4.0), which permits unrestricted use, distribution, and reproduction in any medium, provided the original author(s) and source are credited

Published by: Canadian Science Publishing

where a prime denotes differentiation with respect to $x$. The friction force is modelled by Coulomb's law as $f=\mu N$, where $\mu$ is the friction coefficient and the normal force is $N=\rho g h A_{1}$. Here $\rho$ is the ice density, $g$ is the acceleration of gravity, $h$ is the ice thickness, and $A_{1}$ is the area 
of contact between the ice and the bed. ${ }^{1}$ By considering a unit width of ice in the longitudinal direction, the friction force due to an element of contact length $d s$ is $d f=\mu P d s$. Further, $P=\eta\left(y_{s}-y\right)$, where $y_{s}$ denotes the ice surface and $\eta$ is a constant. Extremizing the friction

$$
f[y(x)]=\mu \eta \int_{x_{1}}^{x_{2}}\left(y_{s}-y\right) \sqrt{1+\left(y^{\prime}\right)^{2}} d x
$$

subject to the constraint (eq. 1.1) leads to

$$
\delta J=\mu \eta \delta \int_{x_{1}}^{x_{2}}\left(y_{s}-y+\lambda\right) \sqrt{1+\left(y^{\prime}\right)^{2}} d x \equiv \delta \int_{x_{1}}^{x_{2}} L=0
$$

where $\lambda$ is a Lagrange multiplier and $L$ is the Lagrangian. The Euler-Lagrange equation

$$
\frac{d}{d x}\left(\frac{\partial L}{\partial y^{\prime}}\right)-\frac{\partial L}{\partial y}=0
$$

yields the ordinary differential equation (ODE) (Hirano and Aniya 1988)

$$
\frac{y_{s}-y+\lambda}{\sqrt{1+\left(y^{\prime}\right)^{2}}}=c_{1}
$$

where $c_{1}$ is a constant. This equation is also obtained by solving the classic catenary problem of mechanics (Goldstein 1980), and therefore, it is not surprising that Hirano and Aniya (1988) obtained catenary solutions of eq. (1.5). Their method and conclusions have been criticized by Harbor (1990; see also Hirano and Aniya 1990; Hirano and Aniya 2005; Morgan 2005). First, the assumptions in the model of Hirano and Aniya (1988) are inconsistent with common assumptions of glaciology (Harbor 1990). Second, friction should be maximized, not minimized (Harbor 1990; although this change does not affect the first-order variational principle, which only requires the friction integral to be extremized). In a reply, Hirano and Aniya (1990) agreed on this point but stood by the validity of application of the variational principle and of their previous result. Further critique (Morgan 2005) pointed out that there is no physical basis for the isoperimetric constraint (eq. 1.1), which should be replaced by the requirement that the area of the cross-section of the glacial valley is fixed. The rationale is that by considering a unit width of ice in the direction of longitudinal flow, the ice volume is thus kept constant (Harbor 1990).

The new Lagrangian constraint in the variational principle leads (Morgan 2005) to the ODE

$$
\left(\frac{y^{\prime}}{y}\right)^{2}=\frac{1}{(\lambda y-C)^{2}}-\frac{1}{y^{2}}
$$

where $y(x)$ is now the ice thickness at transverse coordinate ${ }^{2} x, \lambda$ is again a Lagrange multiplier, and $C$ is a constant, with $\lambda>1$ and $C>0$ required to have a smooth symmetric solution $y(x)$ on the interval $\left[-x_{0}, x_{0}\right]$ with $y^{\prime}(0)=0$ (Morgan 2005). Equation (1.6) is adopted in the rest of this work to describe the transverse profiles of glacial valleys. An exact solution of eq. (1.6) was provided by Morgan (2005):

$$
\left(\lambda^{2}-1\right)|x|=C \lambda \sqrt{1-w^{2}}+C \arccos w
$$

\footnotetext{
${ }^{1}$ If present, water pressure between the glacier and its bed is treated as constant and does not contribute to the variational principle (Hirano and Aniya 1988).

${ }^{2}$ The valley profile is now $z(x)=y_{s}-y(x)$ (cf. Figure 1 of Morgan 2005).
} 
where

$$
\begin{gathered}
w=\left(\frac{\lambda^{2}-1}{C}\right) y-\lambda \\
-\frac{1}{\lambda} \leq w \leq 1, \frac{C}{\lambda} \leq y \leq \frac{C}{\lambda-1}
\end{gathered}
$$

Another formal solution of eq. (1.6) for $\lambda^{2}<1$ has been given recently by Chen et al. (2015a):

$$
\pm \frac{\left(1-\lambda^{2}\right)^{3 / 2}}{|D|} x=\lambda \sqrt{w^{2}-1}+\ln \left|w+\sqrt{w^{2}-1}\right|+D
$$

where $D$ is an integration constant and $|w|>1$. For $C=0$ and $|\lambda|<1$, the solutions are linear.

The constraint of fixed cross-sectional area of the valley may perhaps seem questionable but no better constraint has been proposed in the literature thus far. The requirement of fixed cross-sectional area is also used in the numerical modelling of the erosion process leading to U-shaped valleys (Seddik et al. 2009). In any case, some constraint must be imposed, because if the friction integral is maximized without constraints, the first-order variation

$$
\delta \int_{x_{1}}^{x_{2}}\left(y_{s}-y\right) \sqrt{1+\left(y^{\prime}\right)^{2}} \equiv \delta \int_{x_{1}}^{x_{2}} L_{0}=0
$$

where the Lagrangian is now

$$
L_{0}\left(y(x), y^{\prime}(x)\right)=\left(y_{s}-y\right) \sqrt{1+\left(y^{\prime}\right)^{2}}
$$

produces an equation that only admits unphysical solutions. In fact, as the Lagrangian (eq. 1.12) does not depend explicitly on $x$, the corresponding Hamiltonian $\mathscr{H}_{0}=p_{y} y^{\prime}-L_{0}$ is conserved, where

$$
p_{y}=\frac{\partial L_{0}}{\partial\left(y^{\prime}\right)}=\frac{\left(y_{s}-y\right) y^{\prime}}{\sqrt{1+\left(y^{\prime}\right)^{2}}}
$$

is the momentum canonically conjugated to $y$. The Euler-Lagrange equation (eq. 1.4) for $L_{0}$ has the first integral

$$
\frac{\left(y_{s}-y\right)\left(y^{\prime}\right)^{2}}{\sqrt{1+\left(y^{\prime}\right)^{2}}}-\left(y_{s}-y\right) \sqrt{1+\left(y^{\prime}\right)^{2}}=C
$$

where $C$ is a constant. Using the variable $\xi \equiv y_{s}-y>0$, eq. (1.14) is equivalent to $C \sqrt{1+\left(\xi^{\prime}\right)^{2}}+\xi=0$, which requires that $C<0$; hence we set $C \equiv-C_{2}$, where $C_{2}>0$ has the dimensions of a length. One obtains

$$
\xi^{\prime}= \pm \sqrt{\frac{\xi^{2}}{C_{2}^{2}}-1}
$$

which requires $\xi \geq C_{2}>0$. All the solutions $\xi(x)$ of eq. (1.15) are unbounded from above and are given by 


$$
y_{s}-y(x)=C_{2}^{2} \mathrm{e}^{\mp \frac{\left(x-x_{0}\right)}{C_{2}}}+\frac{\mathrm{e}^{ \pm \frac{\left(x-x_{0}\right)}{C_{2}}}}{4}
$$

(with $x_{0}$ another integration constant) and require $|x| \geq C_{2}$, which does not describe a valley geometry. Therefore, some Lagrangian constraints must be imposed when extremizing the friction integral $f[y(x)]$.

The fact that eq. (1.6) has an analogue in point particle mechanics was missed in the glaciology literature, whereas the fact that it has an analogue in the Friedmann equation of cosmology was recently noted in passing by Chen et al. (2015a, 2015b). Equation (1.6) is a special case of Friedmann-type equations, which are of fundamental importance in cosmology. A mathematical peculiarity of this type of equations demonstrated by Chen et al. (2015b) is that the graphs of all the solutions (in our case, of the transverse valley profiles $y(x))$ are roulettes. A roulette is the locus of a point that lies on, or inside, a curve that rolls without slipping along a straight line.

Our goal is to explore the analogues of the ODE (eq. 1.6) in point particle mechanics and in cosmology, obtaining insight into the properties of this equation and establishing the basics of the formal analogies for future reference. We uncover a type of cosmological singularity, which was studied recently (Bouhmadi-López et al. 2008) for spatially flat universes in the now abundant literature on cosmological singularities (Wald 1984; Barrow et al. 1986; Caldwell 2002; Shtanov and Sahni 2002; Barrow 2004; Nojiri et al. 2005; Dabrowski et al. 2007; Fernandez-Jambrina 2007; Dabrowski and Denkiewicz 2009; Frampton et al. 2011; Bouhmadi-López et al. 2015; Beltran Jimenez et al. 2016).

\section{Particle mechanics analogues of glacial valley cross-profiles}

Following Morgan (2005), we assume $C>0$ (but see the discussion below). The ODE (eq. 1.6) can be rewritten as

$$
\frac{\left(y^{\prime}\right)^{2}}{2}+V(y)=E
$$

where $\left(y^{\prime}\right)^{2} / 2$ is the kinetic energy of a particle of unit mass in one-dimensional motion if $x$ and $y(x)$ are the analogues of time and position, respectively, whereas

$$
V(y) \equiv \frac{-y^{2}}{2(\lambda y-C)^{2}}
$$

is an effective potential energy and $E=-1 / 2$ is the total mechanical energy of the particle. Newton's second law $y^{\prime \prime}=-d V / d y$ then rules the motion of the particle and eq. (2.1) is a first integral corresponding to conservation of energy (with the value $E=-1 / 2$ ). The possible motions $y(x)$ are candidate analogues of the cross-profiles of glacial valleys. A qualitative understanding of the motion can be obtained from the potential $V(y)$ and its intersections with the horizontal line $E=-1 / 2$ (Weierstrass approach; Bochicchio and Laserra 2007; Destrade et al. 2007; Bochicchio et al. 2011).

It is $V(y)<0 \forall y \neq 0, V(0)=0, V(y) \rightarrow-1 /\left(2 \lambda^{2}\right)$ as $y \rightarrow \pm \infty$, there is a vertical asymptote $y=C / \lambda>0$, and $V^{\prime}(y)=C y /(\lambda y-C)^{3}$, therefore the function $V(y)$ increases for $y<0$ and for $y>C / \lambda$, decreases

\footnotetext{
${ }^{3} \mathrm{~A}$ more general definition is that the curve rolls without slipping along another curve but, for Friedmann-type equations, the latter is taken to be a straight line (Chen et al. 2015b).
} 


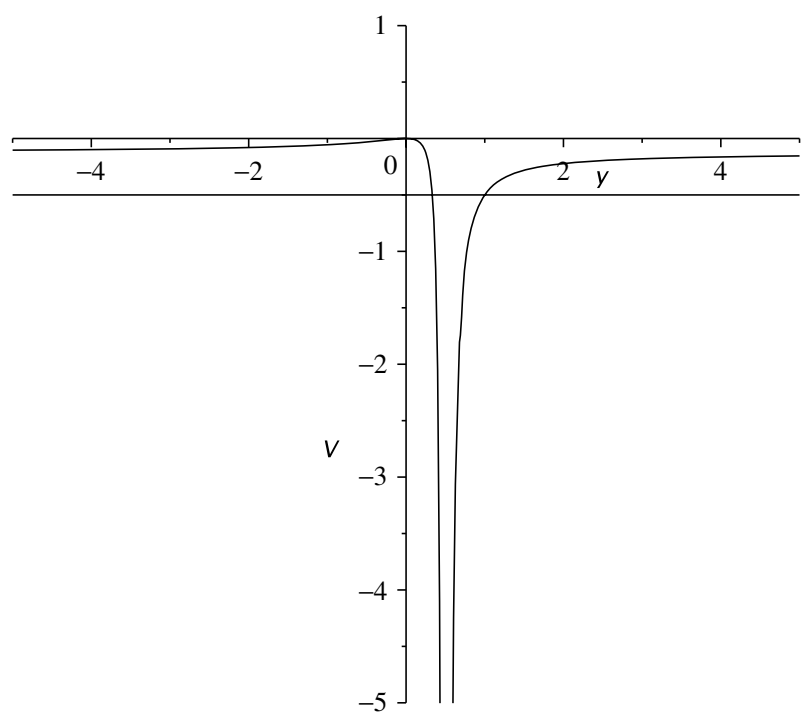

Fig. 1. The potential $V(y)$ intersecting the horizontal line of constant energy $E=-1 / 2$. (The parameter values $\lambda=2$ and $C=1$ are chosen for illustration.)

for $0<y<C / \lambda$, and is maximum at $y=0$ (Fig. 1). We look for regions of bounded motions $y(x)>0$ corresponding to finite ice thickness. We restrict to the situation $C>0, \lambda>0$ for which the vertical asymptote $y=C / \lambda$ of $V(y)$ lies in the $y>0$ region. However, eq. (1.6) and the potential $V(y)$ are invariant under the exchange $(C, \lambda) \rightarrow(-C,-\lambda)$.

\subsection{The case $\lambda>1$}

If $E=-1 / 2<-1 /\left(2 \lambda^{2}\right)$, corresponding to $|\lambda|>1$ (and we take $\lambda>1$ here), the horizontal line $E=-1 / 2$ lies below the horizontal asymptote of $V(y)$ (Fig. 1). There are two regions corresponding to bounded motions $y(x)>0$ (we ignore the region $y<0$ as it is meaningless for the glacial valley problem). The first such region is

$$
0<y_{1} \leq y(x)<\frac{C}{\lambda}
$$

whereas the second region is

$$
\frac{C}{\lambda}<y(x) \leq y_{2}
$$

where $y_{1,2}$ are turning points. The condition $\lambda>1$ for the bounded solutions of Morgan (2005) now receives a graphical interpretation. The particle cannot attain the position $y=C / \lambda$ where the potential diverges and is therefore confined to either one of the regions (eqs. 2.3 or 2.4). The turning points $y_{1,2}$ are the roots of the equation $\left(\lambda^{2}-1\right) y^{2}-2 \lambda C y+C^{2}=0$, or

$$
y_{1,2}=\frac{C}{\lambda \pm 1}
$$

The range $C / \lambda<y(x) \leq C /(\lambda-1)$ reproduces the condition (eq. 1.9) reported by Morgan (2005), whereas the range $C /(\lambda+1) \leq y(x)<C / \lambda$ does not appear in the analysis of this reference, which is therefore augmented by the graphical analogy with mechanics.

\footnotetext{
${ }^{4}$ The turning points correspond to zero slope of the valley profile $y(x)$, therefore to its lowest point.
} 


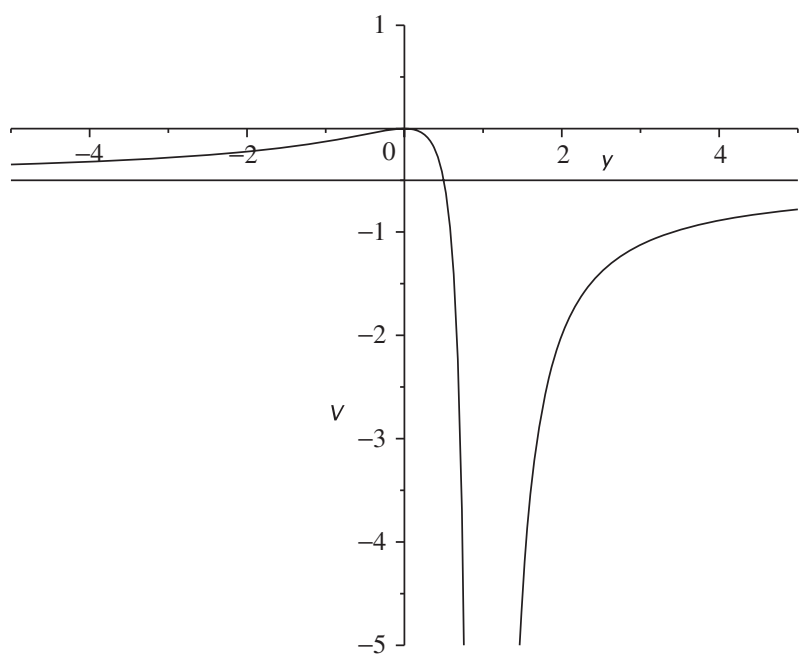

Fig. 2. The potential $V(y)$ for $\lambda=1$. The horizontal line $E=-1 / 2$ intersects the graph of $V(y)$ only once for $y>0$ and there is a region, bounded by this intersection and by the vertical asymptote $y=C / \lambda$, which describes bounded motion. (The parameter value $C=1$ is used in this plot.)

\subsection{The case $\lambda=1$}

If $E=-1 / 2=-1 /\left(2 \lambda^{2}\right)$, corresponding to $\lambda=1$, there is only unbounded motion in the region $y>C / \lambda$, but there is a region of bounded motion $y_{3}=C / 2 \leq y(x)<C / \lambda$, which can make sense as the transverse profile of a glacial valley (Fig. 2). As $\lambda \rightarrow 1^{+}$, one of the turning points (eq. 2.5) is pushed to infinity and effectively disappears, leaving a single turning point $y_{3}=C / 2$.

\subsection{The case $0<\lambda<1$}

If $-1 /\left(2 \lambda^{2}\right)<E=-1 / 2$, corresponding to $\lambda<1$, the horizontal line of constant energy lies above the horizontal asymptote of $V(y)$ and intersects the graph of $V(y)$ only once in the region $y>0$. There is a region of bounded motion $y_{4} \leq y(x)<C / \lambda$. The situation is qualitatively similar to the $\lambda=1$ case. The turning point $y_{4}=C /(1-\lambda)$ lies in the $y>0$ region, whereas the second turning point $y_{5}=-C /$ $(1+\lambda)$ lies in the uninteresting region $y<0$. The analytic solution (eq. 1.10) of eq. (1.6) found by Chen et al. (2015a) belongs to this situation.

\subsection{The case $\lambda<0$}

Let us comment on the second condition $C>0$ of Morgan (2005) and assumed at the beginning of this section. Given the symmetry of eq. (1.6), the situation $C<0$ and $\lambda>0$ is equivalent to $C>0$ and $\lambda<0$, which we discuss here. In this case, the vertical asymptote $y=C / \lambda$ of $V(y)$ lies in the $y<0$ region, and $V^{\prime}(y)$ is negative for $y<C / \lambda<0$ and $y>0$ and is positive for $C / \lambda<y<0$. The graph of $V(y)$ is shown in Fig. 3. If $E=-1 / 2>-1 /\left(2 \lambda^{2}\right)$ (corresponding to $-1<\lambda<0$ ), there is only one intersection $y_{6}=C /(1+\lambda)$ in the $y>0$ region and there are no bounded motions.

In the remaining case $C=0$, eq. (1.6) reduces to $\left(y^{\prime}\right)^{2}=-1+1 / \lambda^{2}$, which has linear solutions corresponding to $\mathrm{V}$-shaped valleys, irrelevant as glacial valley cross-profiles except perhaps as initial conditions (Seddik et al. 2009). This is why we assumed that $C>0$ and we restrict to using this range of $C$ in the rest of this work.

\subsection{Analogue of the no-constraint equation}

In the mechanical analogy, the incorrect eq. (1.15), which would be obtained by extremizing friction without any constraint, can be rewritten as the integral of motion $\left(\xi^{\prime}\right)^{2} / 2+U(\xi)=E$, where 




Fig. 3. The potential $V(y)$ for negative $C$. In this case, the motion is forbidden in the $y>0$ region if $E=-1 / 2 \leq-1 /\left(2 \lambda^{2}\right)$. If $-1<\lambda<0$, the line $E=-1 / 2$ intersects the graph of $V(y)$ only at $y_{6}=C /(1+\lambda)$ in the $y>0$ region and there are no bounded motions. (The parameter values $\lambda=2$ and $C=-1$ are chosen for illustration.)

$E=-1 / 2$ and the potential energy $U(\xi)=-\xi^{2} /\left(2 C_{2}^{2}\right)$ describes an inverted harmonic oscillator. All trajectories (except for the unstable equilibrium position $\xi \equiv 0$, which is meaningless in the original geophysical problem) are unbounded and unphysical.

\section{The universe in a glacial valley}

Relativistic cosmology (Wald 1984; Liddle 2003; Carroll 2004) is obtained by assuming that the fourdimensional spacetime of general relativity is spatially homogeneous and isotropic about every point of three-space, which leads uniquely to the Friedmann-Lemaître-Robertson-Walker (FLRW) line element (Wald 1984; Liddle 2003; Carroll 2004)

$$
d s^{2}=-d t^{2}+a^{2}(t)\left[\frac{d r^{2}}{1-k r^{2}}+r^{2}\left(d \theta^{2}+\sin ^{2} \theta d \varphi^{2}\right)\right]
$$

in comoving polar coordinates $(t, r, \theta, \varphi)$, where the curvature index is normalized to $k=0, \pm 1$, in units in which the speed of light $c$ is unity. The matter content of the universe is assumed to be in the form of a single perfect fluid with energy density $\rho$ and pressure $P$. The functions $a(t), \rho(t)$, and $P(t)$ satisfy the Einstein-Friedmann ODEs (Wald 1984; Liddle 2003; Carroll 2004)

$$
\begin{aligned}
H^{2} & \equiv \frac{\dot{a}^{2}}{a^{2}}=\frac{8 \pi G}{3} \rho-\frac{k}{a^{2}} \\
\frac{\ddot{a}}{a} & =-\frac{4 \pi G}{3}(\rho+3 P)
\end{aligned}
$$

where an overdot denotes differentiation with respect to $t$ and $H(t) \equiv \dot{a} / a$ is the Hubble function, and $G$ is Newton's constant. A third convenient (but not independent) equation expressing covariant conservation of energy is (Wald 1984; Liddle 2003; Carroll 2004) 


$$
\dot{\rho}+3 H(P+\rho)=0
$$

The Friedmann equation (eq. 3.2) is the analogue in cosmology of eq. (1.6) describing the ice thickness in glacial valley transverse profiles. The analogy was recently noted, but not pursued, by Chen et al. (2015b). The most straightforward identification between eqs. (1.6) and (3.2) is achieved by setting $k=1$, which corresponds to a closed universe. Then the cosmological analogue of eq. (1.6)

$$
\frac{\dot{a}^{2}}{a^{2}}=\frac{1}{(\lambda a-C)^{2}}-\frac{1}{a^{2}}
$$

can be rewritten as

$$
\frac{\dot{a}^{2}}{a^{2}}=\frac{8 \pi G}{3} \frac{\rho_{0}}{\left(a-a_{0}\right)^{2}}-\frac{1}{a^{2}}
$$

where

$$
\rho_{0}=\frac{3}{8 \pi G \lambda^{2}}, a_{0}=\frac{C}{\lambda}
$$

are positive constants. Equation (3.2) gives the energy density of the analogue cosmic fluid as

$$
\rho(t)=\frac{\rho_{0}}{\left(a-a_{0}\right)^{2}}
$$

We stress that we study the analogy with possible theoretical models of the future universe dominated by an exotic dark energy, which abound in the literature, and not the actual universe at the present time, which is indeed dominated by dark energy but also contains a non-negligible proportion of dark matter. Other details of the present universe cannot be reproduced by a single equation in the analogy with glacial valley profiles.

Two properties of the density $\rho(t)$ are relevant. First, the energy density is always positive, which is expected of "reasonable" forms of matter but is not at all guaranteed in any formal analogy. Second, the density diverges if $a \rightarrow a_{0}$, corresponding to a spacetime singularity.

"Reasonable" matter in general relativity is supposed to satisfy energy conditions that prohibit negative energy densities and energy flows faster than light (Wald 1984; Liddle 2003; Carroll 2004). When applied to a perfect fluid, the energy conditions are the weak energy condition $(\rho \geq 0$ and $\rho+$ $P \geq 0)$, the strong energy condition $(\rho+P \geq 0$ and $\rho+3 P \geq 0)$, the null energy condition $(\rho+P \geq 0)$ and the dominant energy condition ( $\rho \geq 0$ and $\rho \geq|P|$; Wald 1984; Liddle 2003; Carroll 2004).

The effective pressure $P$ of the analogue cosmic fluid is deduced by imposing eq. (3.4), which yields

$$
P=\frac{2 \rho_{0} a}{3\left(a-a_{0}\right)^{3}}-\frac{\rho_{0}}{\left(a-a_{0}\right)^{2}}
$$

and can be rewritten as

$$
P=-\frac{\rho}{3} \pm \frac{2 a_{0}}{3 \sqrt{\rho_{0}}} \rho^{3 / 2}
$$

where the upper sign applies when $a>a_{0}$ and the lower sign when $a<a_{0}$. Using eqs. (3.8)-(3.10), one concludes easily that for $a>a_{0}$, the weak, strong, and null energy conditions are always satisfied and 
the dominant energy condition is satisfied only when $a \geq 3 a_{0} / 2$. When $a<a_{0}$, all the energy conditions are violated. Equations of state of the cosmic fluid corresponding to the lower sign in eq. (3.10) and violating the weak energy condition have been discussed by Barrow (2004). Equations of state of the cosmic fluid of the form $P=\sum_{k=1}^{m} c_{k} \rho_{(k)}^{k}$ have been studied by Chen et al. (2015a, 2015b), Stefancic (2005), Frampton et al. (2011) and Bouhmadi-López et al. (2015). Quadratic equations of state, in particular, have been the subject of further attention (Nojiri and Odintsov 2004, 2005; Ananda and Bruni 2006a, 2006b; Capozziello et al. 2006; Silva e Costa 2009). Pressures depending on fractional powers of the density have been studied by Nojiri et al. (2005). As discussed below, they give rise to a peculiar type of singularity. Although traditional cosmology and relativity textbooks report only linear barotropic equations of state $P=P_{0}+A \rho$, following the discovery of the acceleration of the universe in 1998, the literature abounds with exotic non-linear equations of state for the dark energy fluid postulated to explain this acceleration. Let us consider now the acceleration equation (eq. 3.3), which, using eq. (3.9), reduces to

$$
\frac{\ddot{a}}{a}=-\frac{a_{0}}{\lambda^{2}\left(a-a_{0}\right)^{3}}
$$

Clearly, the universe is accelerating if $a<a_{0}$ (corresponding to $\rho+3 P<0$ ) and decelerating if $a>a_{0}$ (corresponding to $\rho+3 P>0$ ). The value $a_{0}$ of the scale factor corresponds to a spacetime singularity. In fact, the Einstein equation $R_{a b}-(1 / 2) g_{a b} R=8 \pi G T_{a b}$ (where $R_{a b}$ is the Ricci tensor) gives $R \equiv g^{a b} R_{a b}=-8 \pi G T$, where $T$ is the trace of the fluid energy momentum tensor (Wald 1984; Liddle 2003; Carroll 2004)

$$
T_{a b}=(P+\rho) u_{a} u_{b}+P g_{a b}
$$

with four-velocity $u^{a}$. The Ricci scalar is, therefore,

$$
R=8 \pi G(\rho-3 P)=\frac{16 \pi G \rho_{0}}{\left(a-a_{0}\right)^{3}}\left(a-2 a_{0}\right)
$$

which diverges in the limit $a \rightarrow a_{0}$ signalling a spacetime singularity. It is not yet clear whether the value $a_{0}$ of the scale factor can actually be approached during the dynamics. To answer this question, note that eq. (3.2) can be written as

$$
a^{2} \geq \lambda^{2}\left(a-a_{0}\right)^{2}
$$

excluding the orbits of the solutions of the dynamical system (eqs. 3.2 and 3.3) from a certain volume of the $(a, \dot{a})$ phase space.

If $a>a_{0}$, the dynamical constraint (eq. 3.14) can be written as $a \leq \lambda a_{0} /(\lambda-1)$. The coefficient of $a_{0}$ is $\lambda /(\lambda-1)=1+1 /(\lambda-1)>1$, and therefore, in this regime we have $a_{0}<a<\lambda a_{0} /(\lambda-1)$; the scale factor $a(t)$ is bounded from above but it can get arbitrarily close to the value $a_{0}$ corresponding to the singularity.

If instead $a<a_{0}$, then the constraint (eq. 3.14) becomes $a \geq \lambda a_{0} /(\lambda+1)$ and one has $0<\lambda a_{0} /(\lambda+1) \leq$ $a<a_{0}$. In both cases, the scale factor is bounded from below by a positive constant (hence one cannot have a Big Bang- or Big Crunch-type singularity corresponding to $a \rightarrow 0$; Wald 1984; Liddle 2003; Carroll 2004) but it can reach the singularity ${ }^{5}$. It corresponds to the fact that $y=0$ (equivalently $a=0$ ) lies in the region of the phase space forbidden by the constraint (eq. 3.14).

\footnotetext{
${ }^{5}$ The fact that one cannot have $y=0$ in the original equation (eq. 1.6) because it implies an imaginary $y^{\prime}$ was
} noted as "a curious feature" by Morgan (2005). 
The boundary values $a=\lambda a_{0} /(\lambda \mp 1)$ are formal solutions of eq. (3.5) obtained by setting $a=$ constant, but they do not satisfy eq. (3.3) (they would be meaningless as analogues of glacial valley profiles).

Consider again the acceleration equation (eq. 3.3): if $a>a_{0}$, then $\ddot{a}<0$ and the curve representing the scale factor has concavity facing downwards. As this curve is continuous, it always decreases and eventually crosses the horizontal line $a=a_{0}$.

Using eq. (3.7), eq. (3.5) is written as

$$
\dot{a}^{2}=\frac{a^{2}}{\lambda^{2}\left(a-a_{0}\right)^{2}}-1 \approx \frac{a_{0}^{2}}{\lambda^{2}\left(a-a_{0}\right)^{2}}
$$

as $a \rightarrow a_{0}$. This asymptotic equation is easily integrated to

$$
a(t) \simeq a_{0} \pm \sqrt{\frac{2 a_{0}}{\lambda}\left|t-t_{0}\right|}
$$

where the integration constant $t_{0}$ has the meaning of time at which the singularity occurs and the positive sign of the square root must be chosen because $a>a_{0}$. This situation constitutes a physically meaningful analogue of glacial valleys because $y$ is the thickness (maximum at $x=0$ and minimum at the valley boundaries; Morgan 2005) and it is interesting in cosmology because it provides an example of a finite-time singularity even when $\rho>0$ and $\rho+P>0$ (i.e., without violating the weak energy condition), an interesting situation discussed by Barrow (2004). Vice versa, if $a<a_{0}$ then $\ddot{a}>0$ and $a(t)$ always increases, eventually crossing the horizontal line $a=a_{0}$. The asymptotic equation (eq. 3.15) now integrates to

$$
a(t) \simeq a_{0}-\sqrt{\frac{2 a_{0}}{\lambda}\left|t-t_{0}\right|}
$$

choosing the negative sign in front of the square root because now $a<a_{0}$. This situation is not a meaningful analogue of a glacial valley cross-profile. The slope of this function becomes infinite where $a(t) \rightarrow a_{0}$, corresponding to a spacetime singularity in the cosmological analogue. In this case, the universe has a minimum size $a_{\min }=\lambda a_{0} /(\lambda+1)$ and it bounces upon reaching it. The bounce occurs when the weak energy condition is violated, which is exactly what is happening here because $\rho+P=2 \rho_{0} a / 3\left(a-a_{0}\right)^{3}<0$. The violation of the weak energy condition signals a very exotic phantom fluid, which causes the universe to accelerate with Hubble function increasing according to $\dot{H}=-4 \pi G(\rho+P)+k / a^{2}>0$ if $k \geq 0$. This superacceleration causes the universe to expand superexponentially and reach a singularity at a finite time. In the standard cosmological literature, the scale factor of a phantom-dominated universe diverges at a finite time in the future at a Big Rip singularity (Caldwell 2002), but here the situation is different because the scale factor stays finite while the Hubble function $H$, energy density $\rho$, pressure $P$, and Ricci scalar $R$ all diverge as $a \rightarrow a_{0}$. This situation corresponds instead to a type of singularity studied recently in spatially flat universes and called a Big Freeze singularity (Bouhmadi-López et al. 2008) or Type III singularity in the classifications of Nojiri et al. (2005) and Bamba et al. (2012). A Big Freeze singularity also appears in cosmology in the context of Palatini $f(R)$ gravity (Borowiec et al. 2012, 2016; Szydlowski et al. 2016; Stachowski et al. 2016). A Big Freeze singularity was not previously reported for positively curved universes. The situation is essentially the same as for spatially flat universes because, as $a \rightarrow a_{0}$ in eq. (3.6), the divergent term proportional to $\left(a-a_{0}\right)^{-2}$ in the energy density dominates over the curvature term $-1 / a^{2}$, which stays finite. Finite-time singularities, including Big Rip (Caldwell 2002) and sudden future singularities (Barrow et al. 1986; 
Barrow 2004), have been the subject of a significant amount of work in cosmology (Shtanov and Sahni 2002; Kofinas et al. 2003; Calcagni 2004; Gorini et al. 2004; Nojiri et al. 2005; Stefancic 2005; Dabrowski et al. 2007; Fernandez-Jambrina 2007; Bouhmadi-López et al. 2008; Dabrowski and Denkiewicz 2009; Frampton et al. 2011; Bamba et al. 2012; Bouhmadi-López et al. 2015; Beltrán Jiménez 2016).

Finally, although not interesting for the original glacial valley problem, the cosmic analogue of eq. (1.6) for $C=0$ (noted also by Chen et al. (2015b)) corresponds to $a_{0}=0$ and

$$
H^{2}=\frac{1}{a^{2}}\left(\frac{1-\lambda^{2}}{\lambda^{2}}\right)
$$

and requires $|\lambda| \leq 1$. Then $\dot{a}=$ constant and the solutions are linear in time and include a static universe with $a=$ constant as a special case.

\section{Discussion}

In glacial morphology studies, researchers content themselves with fitting data of glacial valley ice thickness with parabolas $y(x)=a x^{2}+b x+c$ (following an early practice initiated by Svensson (1959), which is not free of critique (Pattyn and Van Huele 1998)). Other fitting curves used include power-law profiles $y(x)=a x^{b}$, possibly with different powers $b$ for each half-profile going from the bottom at $x=0$ to each valley side. There is a deep disconnect between theory and practice here. As a parabola is just the second-order Taylor expansion of any sufficiently regular function with a minimum, which could solve infinitely many ODEs, data fitting with parabolas is of no help when one attempts to test models and to discriminate among theoretical approaches to the problem of the cross-sectional profiles of glacial valleys, which predict different ODEs for the profile $y(x)$. In this sense, fitting parabolas or power-laws and determining best-fit parameters is deeply unsatisfactory from the theoretical point of view and does not contribute to understanding the mechanism that generated these profiles.

Here we focused on the variational principle approach to the problem of glacial valley erosion, in the form given by Morgan (2005). The resulting equation (eq. 1.6) for the ice thickness $y(x)$ has analogues in point particle mechanics and in cosmology, which we explored in detail. These analogies contribute to a better understanding of this ODE, its solutions, and the conditions (on the parameters $\lambda$ and $C$ ) for their physical viability. The previous analysis of Morgan (2005) is augmented by the graphical study of the effective potential $V(y)$ in the mechanical analogy. The problem of glacial valley profiles provides also an interesting example of a finite-time singularity in current theoretical cosmological models without violating the weak energy condition. The finite-time singularity is caused by the peculiar effective equation of state (eq. 3.10), which falls into the broader category $P=w \rho-\alpha \rho^{m}$ (with $w, \alpha$, and $m$ constants), which has been the subject of wide interest in cosmology ${ }^{6}$ (Shtanov and Sahni 2002; Kofinas et al. 2003; Barrow 2004; Calcagni 2004; Gorini et al. 2004; Nojiri et al. 2005; Stefancic 2005; Frampton et al. 2011; Bouhmadi-López et al. 2015).

The present work also highlights two open problems in glaciology. First, the friction of glacier ice against the valley walls and floor is unlikely to be described purely by Coulomb's law, but it should include viscous friction that depends on the velocity (a friction model quadratic in

\footnotetext{
${ }^{6}$ Note that in the work of Kofinas et al. (2003) the conditions are similar to those of our analogue model only in the far future.
} 
the velocity is used, for example, in the numerical work of Seddik et al. (2009)). Second, the numerical analyses of the formation of glacial valleys ignore the variational approach (but not its Lagrangian constraint) and should be compared with it. These issues will be revisited in future work.

\section{Acknowledgements}

This work is supported by the Natural Sciences and Engineering Research Council of Canada.

\section{Author contributions}

Conceived and designed the study: VF. Contributed resources: VF. Drafted or revised the manuscript: VF, AMC.

\section{Competing interests}

VF is currently serving as a Subject Editor for FACETS, but was not involved in review or editorial decisions regarding this manuscript.

\section{Data accessibility statement}

All relevant data are within the paper.

\section{References}

Ananda KN, and Bruni M. 2006a. Cosmological dynamics and dark energy with a quadratic equation of state: anisotropic models, large-scale perturbations and cosmological singularities. Physical Review D, 74: 023524. doi:10.1103/PhysRevD.74.023524.

Ananda KN, and Bruni M. 2006b. Cosmological dynamics and dark energy with nonlinear equation of state: a quadratic model. Physical Review D, 74: 023523. doi:10.1103/PhysRevD. 74.023523 .

Bamba K, Capozziello S, Nojiri S, and Odintsov SD. 2012. Dark energy cosmology: the equivalent description via different theoretical models and cosmography tests. Astrophysics \& Space Science, 342: 155-228. doi:10.1007/s10509-012-1181-8.

Barrow JD. 2004. Sudden future singularities. Classical \& Quantum Gravity, 21: L79-L82. doi:10.1088/0264-9381/21/11/L03.

Barrow JD, Galloway G, and Tipler FJT. 1986. The closed-universe recollapse conjecture. Monthly Notices of the Royal Astronomical Society, 223: 835-844. doi:10.1093/mnras/223.4.835.

Beltrán Jiménez J, Rubiera-Garcia D, Sáez-Gómez D, and Salzano V. 2016. Q-singularities. preprint arXiv:1607.06389.

Bochicchio I, Capozziello S, and Laserra E. 2011. The Weierstrass criterion and the LemaîtreTolman-Bondi models with cosmological constant $\Lambda$. International Journal of Geometrical Methods of Modern Physics, 8: 1653-1666. doi:10.1142/S0219887811005865.

Bochicchio I, and Laserra E. 2007. On the mechanical analogy between the relativistic evolution of a spherical dust universe and the classical motion of falling bodies. Journal of Interdisciplinary Mathematics, 10: 747-755. doi:10.1080/09720502.2007.10700530. 
Borowiec A, Kamionka M, Kurek A, and Szydlowski M. 2012. Cosmic acceleration from modified gravity with Palatini formalism. Journal of Cosmology and Astroparticle Physics, 2012: 27. doi:10.1088/1475-7516/2012/02/027.

Borowiec A, Stachowski A, Szydlowski M, and Wojnar A. 2016. Inflationary cosmology with Chaplygin gas in Palatini formalism. Journal of Cosmology and Astroparticle Physics, 2016(01): 40. doi:10.1088/1475-7516/2016/01/040.

Bouhmadi-López M, Errahmani A, Martin-Moruno P, Ouali T, and Tavakoli Y. 2015. The little sibling of the big rip singularity. International Journal of Modern Physics D, 24: 1550078/1-20. doi:10.1142/S0218271815500789.

Bouhmadi-López M, Gonzalez-Diaz PF, and Martin-Moruno P. 2008. Worse than a big rip? Physics Letters B, 659: 1-5. doi:10.1016/j.physletb.2007.10.079.

Calcagni G. 2004. Slow-roll parameters in braneworld cosmologies. Physical Review D, 69: 103508. doi:10.1103/PhysRevD.69.103508.

Caldwell RR. 2002. A phantom menace? Cosmological consequences of a dark energy component with super-negative equation of state. Physics Letters B, 545: 23-29. doi:10.1016/S0370-2693(02) 02589-3.

Campbell JF. 1865. Frost and fire. J.B. Lippincott, Philadelphia, Pennsylvania.

Capozziello S, Cardone VF, Elizalde E, Nojiri S, and Odintsov SD. 2006. Observational constraints on dark energy with generalized equations of state. Physical Review D, 73: 043512. doi:10.1103/ PhysRevD.73.043512.

Carroll SM. 2004. Spacetime and geometry: an introduction to general relativity. Addison Wesley, San Francisco, California.

Chen A, Gibbons GW, and Yang Y. 2015a. Explicit integration of Friedmann's equation with nonlinear equations of state. Journal of Cosmology and Astroparticle Physics, 2015: 020. doi:10.1088/ $1475-7516 / 2015 / 05 / 020$.

Chen A, Gibbons GW, and Yang Y. 2015b. Friedmann-Lemaitre cosmologies via roulettes and other analytic methods. Journal of Cosmology and Astroparticle Physics, 2015: 056. doi:10.1088/1475-7516/ 2015/10/056.

Dabrowski MP, and Denkiewicz T. 2009. Barotropic index $w$-singularities in cosmology. Physical Review D, 79: 063521. doi:10.1103/PhysRevD.79.063521.

Dabrowski MP, Denkiewicz T, and Hendry MA. 2007. How far is it to a sudden future singularity of pressure? Physical Review D, 75: 123524. doi:10.1103/PhysRevD.75.123524.

Destrade M, Gaeta G, and Saccomandi G. 2007. Weierstrass's criterion and compact solitary waves. Physical Review E, 75: 047601. doi:10.1103/PhysRevE.75.047601.

Fernández-Jambrina L. 2007. Hidden past of dark energy cosmological models. Physics Letters B, 656: 9-14. doi:10.1016/j.physletb.2007.08.091.

Frampton PH, Ludwick KJ, and Scherrer RJ. 2011. The little rip. Physical Review D, 84: 063003/1-5. doi:10.1103/PhysRevD.84.063003. 
Goldstein H. 1980. Classical mechanics. Addison-Wesley, Reading, Massachusetts.

Gorini V, Kamenshchik A, Moschella U, and Pasquier V. 2004. Tachyons, scalar fields and cosmology. Physical Review D, 69: 123512. doi:10.1103/PhysRevD.69.123512.

Harbor J. 1990. A discussion of Hirano and Aniya's $(1988,1989)$ explanation of glacial-valley cross profile development. Earth Surface Processes and Landforms, 15: 369-377. doi:10.1002/ esp.3290150408.

Harbor JM. 1995. Development of glacial-valley cross sections under conditions of spatially variable resistance to erosion. Geomorphology, 14: 99-107. doi:10.1016/0169-555X(95) 00051-1.

Hirano M, and Aniya M. 1988. A rational explanation of cross-profile morphology for glacial valleys and of glacial valley development. Earth Surface Processes and Landforms, 13: 707-716. doi:10.1002/ esp.3290130805.

Hirano M, and Aniya M. 1990. A reply to "a discussion of Hirano and Aniya's $(1988,1989)$ explanation of glacial-valley cross profile development" by Jonathan M. Harbor. Earth Surface Processes and Landforms, 15: 379-381. doi:10.1002/esp.3290150409.

Hirano M, and Aniya M. 2005. Response to Morgan's comment. Earth Surface Processes and Landforms, 30: 515. doi:10.1002/esp.1221.

Kofinas G, Maartens R, and Papantonopoulos E. 2003. Brane cosmology with curvature corrections. Journal of High Energy Physics, 2003: 066. doi:10.1088/1126-6708/2003/10/066.

Liddle A. 2003. An introduction to modern cosmology. Wiley, Chichester.

McGee WJ. 1894. Glacial canons. Journal of Geology, 2: 350-364. doi:10.1086/606974.

Morgan F. 2005. A note on cross-profile morphology for glacial valleys. Earth Surface Processes and Landforms, 30: 513-514. doi:10.1002/esp.1220.

Nojiri S, and Odintsov SD. 2004. Final state and thermodynamics of a dark energy universe. Physical Review D, 70: 103522. doi:10.1103/PhysRevD.70.103522.

Nojiri S, and Odintsov SD. 2005. Inhomogeneous equation of state of the universe: phantom era, future singularity, and crossing the phantom barrier. Physical Review D, 72: 023003. doi:10.1103/ PhysRevD.72.023003.

Nojiri S, Odintsov SD, and Tsujikawa S. 2005. Properties of singularities in the (phantom) dark energy universe. Physical Review D, 71: 063004/1-16. doi:10.1103/PhysRevD.71.063004.

Pattyn F, and Van Huele W. 1998. Power law or power flaw? Earth Surface Processes and Landforms, 23: 761-767. doi:10.1002/(SICI)1096-9837(199808)23:8<761::AID-ESP892>3.0. $\mathrm{CO} ; 2-\mathrm{K}$.

Seddik H, Greve R, Sugiyama S, and Naruse R. 2009. Numerical simulation of the evolution of glacial valley cross sections. preprint arXiv:0901.1177.

Shtanov Y, and Sahni V. 2002. New cosmological singularities in braneworld models. Classical \& Quantum Gravity, 19: L101-L107. doi:10.1088/0264-9381/19/11/102. 
Silva e Costa S. 2009. An entirely analytical cosmological model. Modern Physics Letters A, 24: 531-540. doi:10.1142/S021773230902845X.

Stachowski A, Szydlowski M, and Borowiec A. 2016. Starobinsky cosmological model in Palatini formalism. preprint arXiv:1608.03196.

Stefancic H. 2005. Expansion around the vacuum equation of state: sudden future singularities and asymptotic behavior. Physical Review D, 71: 084024/1-9. doi:10.1103/physrevd.71.084024.

Svensson H. 1959. Is the cross-section of a glacial valley a parabola? Journal of Glaciology, 3: 362-363.

Szydlowski M, Stachowski A, Borowiec A, and Wojnar A. 2016. Do sewn up singularities falsify the Palatini cosmology? The European Physical Journal C, 76: 567. doi:10.1140/epjc/s10052-016-4426-9.

Wald RM. 1984. General relativity. Chicago University Press, Chicago, Illinois.

Yang SH, and Shi YL. 2015. Three-dimensional numerical simulation of glacial trough forming process. Science China (Earth Sciences), 58: 1656-1668. doi:10.1007/s11430-015-5120-8. 